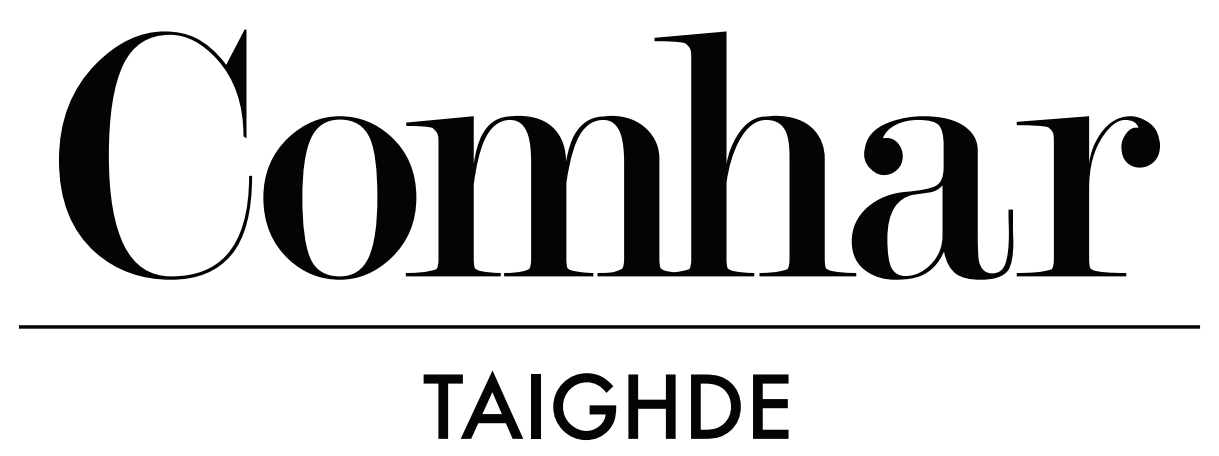

\title{
EAGRÁN 3
}

Deireadh Fómhair 2017

\section{Léirmheas}

An Piarsach agus 1916: Briathar, Beart agus Oidhreacht

Dáta foilsithe:

19 Deireadh Fómhair 2017

\section{Cóipcheart:}

(c) Cathal Ó Háinle, 2017

Seoladh gréasáin:

https://comhartaighde.ie/eagrain/3/ohainle/
Léirmheastóir:

Cathal Ó Háinle

\section{Comhfhreagras:}

cohainle@tcd.ie

Seoladh seasmhach (DOI):

https://doi.org/10.18669/ct.2017.13

Arna fhoilsiú ag COMHAR Teoranta le cabhair deontais i gcomhair tograí Gaeilge a d'íoc an tÚdarás um Ard-Oideachas trí Choláiste na hOllscoile, Corcaigh. Gabhann COMHAR buíochas le hAcadamh Ríoga na hÉireann as a dtacaíocht leis an bhfiontar seo.

\section{comhartaighde.ie}

ISSN: 2009-8626 


\title{
An Piarsach agus 1916: Briathar, Beart agus Oidhreacht
}

\author{
Gearóid Ó Tuathaigh (eag.) \\ Cló lar-Chonnacht (2016) | 321 Ich | ISBN: 978-1-78444-139-5 | €15
}

Is é atá sa leabhar seo díolaim aistí atá bunaithe ar léachtaí cuimhneacháin ar Éirí Amach 1916 a craoladh ar RTÉ Raidió na Gaeltachta sa bhliain 2016. Ba é stiúrthóir Chló Iar-Chonnacht, Micheál Ó Conghaile, a mhol go gcuirfí an tsraith sin léachtaí á dtabhairt agus a d'fhoilsigh iad mar leabhar ina dhiaidh sin. Dhá aiste dhéag atá sa leabhar, agus iad ar fad eolach spéisiúil. Tá cuid de na haistí níos faide ná an meán, roinnt acu cuid mhaith níos faide, aiste Ghearóid Denvir go háirithe, agus tá nótaí mínithe agus tagartha ag gabháil leo uile, seachas aiste Alan Titley a bhfuil alt gearr, ina dtráchtann sé ar a chuid foinsí, ag gabháil léi. Léirítear agus pléitear gnéithe de mheon, de phearsantacht, de shaothar, de ghníomhartha agus de shaol an Phiarsaigh i ngach ceann de na haistí, ach amháin an ceann deiridh ina bpléann eagarthóir an leabhair, Gearóid Ó Tuathaigh, an tráchtaireacht atá déanta air ó aimsir a bháis i leith.

Agus a rá nach raibh sé ach sé bliana tríochad nuair a cuireadh chun báis é, is éachtach an méid a chuir an Piarsach i gcrích mar phearsa lárnach de chuid Athbheochan na Gaeilge, mar cheannródaí i bhforbairt nualitríocht na Gaeilge, mar iriseoir, mar eagarthóir irisí agus mar scríbhneoir cruthaitheach, mar oideachasóir, agus mar dhuine de cheannairí Éirí Amach na Cásca 1916. Ach is léir gur ógánach seanchríonna $a b$ ea é a thosaigh agus é an-óg ag cur spéis ghníomhach sa dúchas Gaelach agus ag féachaint leis an spéis chéanna a mhúscailt ina chomhaoiseanna. Nuair nach raibh sé ach i rang na Meánteistiméireachta thug sé cuairt ar an Leabharlann Náisiúnta mar ar léigh sé dhá bhailiúchán béaloidis le Dubhghlas de hÍde, Leabhar Sgeulaigheachta (1889) agus Cois na Teineadh (1892). Agus nuair nach raibh sé fós naoi mbliana déag d'aois cláraíodh é mar bhall de Chonradh na Gaeilge, bhunaigh sé cumann ar ar bhaist sé an teideal uaillmhianach 'New Ireland Literary Society,' agus i ngiorracht aimsire bhí sé tar éis trí léacht a thabhairt do bhaill an chumainn sin agus na léachtaí sin a fhoilsiú mar leabhrán $(23,26)$.

Deir Róisín Ní Ghairbhí sa chéad aiste sa leabhar seo,'Pádraic Mac Piarais 1879-1916: Teacht in Inmhe,' gur léir go raibh bród ar an bPiarsach 'as cuimhne a mhuintire [ar thaobh a mháthar] ar a n-oidhreacht Ghaelach' (21). D'fhoghlaim sé mórán focal Gaeilge óna uncail Christy Brady agus is minic a chuala sé a aintín Margaret ag rá amhrán Gaeilge, agus ag reic seanchais agus scéalta, ionas gur thug sé uirthi "the woman to whom I owe all my enthusiasms"'(22). Más ó na Brádaigh a fuair sé a spéis ina dhúchas Gaelach agus a chion air, is óna athair a fuair sé an aigne cheisteach neamhspleách, an meon ealaíonta agus bua na fiontraíochta (15-18) a chuir ar a chumas saothrú chun an nasc leis an dúchas Gaelach a athcheangal agus chun a áitiú ar phobal na hÉireann gur cheart tús áite a thabhairt don dúchas sin i saol na tíre.

Comhthoghadh an Piarsach ina bhall d'Ardchoiste an Chonartha i 1898, ceapadh é ina rúnaí ar Choiste na bhFoilseachán i 1900 agus i 1903 ceapadh é ina eagarthóir ar An Claidheamb Soluis, irisleabhar an Chonartha. Thuig sé gurb é gnó na hirise teagasc agus aidhmeanna an Chonartha a fhógairt ach, mar a léiríonn Regina Uí Chollatáin ina haiste 'An Piarsach agus Iriseoireacht na Gaeilge: Iriseoir Intleachtúil Victeoiriach agus Réabhlóidí Machnamhach,' déiligh sé an tsaoirse lena thuiscintí féin ina dtaobh sin a chraobhscaoileadh go neamhspleách údarásach (46). I dtús báire dúirt sé go mbeadh sé d'aidhm ag 
an iris "a consecutive and adequate record of the home and foreign history of the week" a thabhairt do chainteoirí dúchais na Gaeilge "in vivid idiomatic Irish" (44), ionas go mbeadh Éire in ann a háit a ghlacadh i measc thíortha an domhain. Tá sé íorónta gur i mBéarla a scríobh sé an méid sin; ach ba í an fhírinne thruamhéalach í nach raibh ach fíorbheagán de phobal na tíre in ann an Ghaeilge a léamh. Dá bharr sin, iris dhátheangach ab ea An Claidheamb Soluis agus is i mBéarla a scríobh an Piarsach cuid anmhór dá chuid aistí agus eagarfhocal inti.

Is léir gur dheacair don Phiarsach a bheith beo gan a bheith i mbun irise tar éis dó éirí as eagarthóireacht An Claidheamb Soluis i 1909 (47). Chuir sé An Macaomb ar bun sa bhliain chéanna sin agus bhí mar eagarthóir uirthi go dtí 1913, agus i Márta na bliana 1912 bhunaigh sé An Barr Buadh.

Ba í aidhm an Chonartha tús áite i saol na hÉireann a bhaint amach don dúchas Gaelach agus don Ghaeilge, ach d'áitigh an Piarsach gur ghá an nasc idir an t-idéal agus an gníomh a neartú trí 'ról gníomhach a ghlacadh i gcur chun cinn Ghaelachas na sochaí ag gach leibhéal agus i ngach institiúid in Éirinn' (44). Chuige sin dhearbhaigh sé a thábhachtaí a bhí an tsaoirse intleachtúil, agus nocht sé go tréamanta in $A n$ Claidheamb Soluis a bharúlacha i dtaobh ionad lárnach na Gaeilge san oideachas, i dtaobh na litríochta, i dtaobh ról na cléire agus na hEaglaise i gcur chun cinn an Ghaelachais, agus i dtaobh gnéithe áirithe den saol sóisialta.

In 'Pádraic Mac Piarais: Oideachasóir', tugann Ciarán Ó Coigligh mórán dréachtaí, go díreach nó go hindíreach, as aistí de chuid an Phiarsaigh in An Claidheamb Soluis faoin oideachas sa bhunscoil, sa mheánscoil, sna coláistí oideachais, i gColáiste Phádraig, Maigh Nuad (an cliarcholáiste) agus san Ollscoil. Bhí na modhanna múinte agus an Clár Dátheangach ar na gnéithe ba thábhachtaí den chur chuige san oideachas, dar leis, agus d'fhoilsigh sé dhá shraith thábhachtacha aistí faoin gcur chuige dátheangach sa Bheilg, rud a raibh eolas maith aige air de bharr na tréimhse a chaith sé i scoileanna na Beilge sa bhliain 1905. D’fháiltigh sé ó chroí roimh an gClár Dátheangach (122), leag creatlach cúrsa amach le haghaidh naíonán i scoil Ghaeltachta (124) agus mhol go mbeadh 'an Béarla mar dhara teanga' $i$ scoileanna na Gaeltachta (133), cé gur admhaigh sé 'nár fhonn leis féin go pearsanta go múinfí Béarla ar bith do ghasúir Éireannacha' (121). An modh díreach $\mathrm{ab}$ ansa leis mar mhodh múinteoireachta, ach is léir go mbeadh deacrachtaí móra ag baint leis sin i bhfianaise an chuntais a thug sé féin ar ionad dearóil na Gaeilge sna coláistí oideachais (132-133).

Caitliceach dílis cráifeach ab ea an Piarsach, ach níor fhág sé sin nach bhféadfadh sé an chléir agus an cliarlathas a cháineadh faoi gan tacú le polasaithe an Chonartha i dtaobh na Gaeilge. Luann Ó Coigligh gur fhógair sé gurbh é an “"non-Irish-speaking priest in an Irish-speaking district" namhaid mór na Gaeilge (138), gur lochtaigh sé John Healy, ArdEaspag Thuama, go géar i ngeall ar an tslí a ndearna sé coilichín paor de phobal na Gaeilge (142-143) agus gur cháin sé údaráis Choláiste Mhaigh Nuad nuair a bhris siad an Dr Micheál Ó hIceadha as a ollúnacht (168) toisc gur ionsaigh sé an cliarlathas mar nár sheas siad leis an nGaeilge a bheith ina hábhar riachtanach le haghaidh an mháithreánaigh san Ollscoil Náisiúnta. Sílim nár mhiste don Choiglíoch léiriú a thabhairt ar chúlra an ábhair a bhí faoi chaibidil ag an bPiarsach i gcásanna áirithe ar a laghad, Bille Ollscoile Birrell (1908), mar shampla.

Bhí taithí phearsanta ag an bPiarsach ar mhúinteoireacht ar an dara leibhéal mar theagascóir i Scoil na mBráithre Críostaí, Rae an Iarthair, agus mar mhúinteoir buan i gColáiste Alexandra, Ardán Phort an Iarla, le linn a bhlianta tosaigh i mbun $A n$ Claidheamb Soluis; ach fuair sé taithí ar riaradh scoile tar éis dó Scoil Éanna a chur ar bun in Cullenswood House, Ráth Maonais, sa bhliain 1908. Bhí éileamh chomh mór sin ar áiteanna sa scoil gur aistrigh sé í go dtí The Hermitage i Ráth Fearnáin i 1910. Theastaigh uaidh 'neamhspleáchas intleachtúil' a chothú sa scoil: bheadh dearcadh Gaelach á chur chun tosaigh, teagasc dátheangach á chleachtadh, tírghrá á chothú, béim ar leith á cur ar an eolaíocht agus ar ábhair nua-aimseartha; agus bheadh carachtar na ndaltaí á mhúnlú agus bheadh baint ag na daltaí le ceapadh an churaclaim. Mar a léiríonn Fionntán de Brún ina aiste 'Pádraic Mac Piarais, Scoil Éanna agus Neamhspleáchas Intleachtúil na hÉireann,' bhain an Piarsach leas mór as ainm agus láthair na scoile chun an stair a leagan mar dhúshraith faoin oideachas a tugadh inti. Bhí baint le The Hermitage ag Wolfe Tone agus ag Robert Emmet, beirt a shaothraigh 
chun neamhspleáchas na hÉireann a bhaint amach. B'ábhar inspioráide don Phiarsach an leanúnachas staire sin, agus bhí stair na scoile a bhunaigh Éanna in Árainn ag teacht 'leis an dúil a bhí [aige] in eiseamláir náisiúnta oideachais a bhunú agus leis an luí a bhí aige le cumhacht an útóipeachais ... mar shlí leis an seanmhúnla tuisceana a bhriseadh agus ord nua a bhunú ina áit' (162). Bhunaigh sé Scoil Éanna 'le hathghreim a fháil ar ... neamhspleáchas intleachtúil [na hÉireann]' (168).

Chun cuidiú le forbairt intleachtúil na ndaltaí i Scoil Éanna thug sé deis dóibh aistí a fhoilsiú in iris na scoile, An Macaomb, le hais aistí na múinteoirí. Agus ar an gcaoi chéanna, chun a samhlaíocht a chothú, chuir sé an drámaíocht chun fónaimh: rinneadh staidéar sa scoil ar dhrámaí le William Shakespeare (104), aistríodh agus léiríodh radharcanna as Hamlet (69), d'fhreastail na daltaí ar dhrámaí san ardchathair, agus léirigh siad drámaí leis an bPiarsach féin agus le húdair Éireannacha eile (104) chomh maith le dráma le Rabindranath Tagore (165). Bhí an ghlóirréim Macghniombartha Chúchulainn ar cheann de na drámaí a chum sé: léiríodh í i mí an Mheithimh 1909 mar cheiliúradh ar dheireadh na chéad scoilbhliana. Gné eile ab ea é sin, agus an dara glóir-réim a chum sé, The Defence of the Ford, d'iarracht an Phiarsaigh leis an 'stair' a leagan mar dhúshraith faoi éiteas na scoile. Bhíodh sé de shíor ag cur Chú Chulainn mar eiseamláir den laochas faoi bhráid na ndaltaí agus é ag iarraidh ath-Chú Chulainn a dhéanamh den uile dhuine díobh, mar a dúirt duine de na daltaí sin, Pádhraic Óg Ó Conaire, ionas, mar a dúirt duine eile díobh, Desmond Ryan, go ndearnadh 'an invisible member of the school staff' den Chú (1919: 83).

Ar an drochuair thit líon na ndaltaí tar éis an aistrithe go Ráth Fearnáin agus bhí na costais a bhain leis an aistriú an-trom, ionas nach raibh an Piarsach in ann iad a ghlanadh. Thug sé a aghaidh ar Mheiriceá i bhFeabhra na bliana 1914 chun sraith léachtaí a thabhairt agus súil aige go n-éireodh leis dóthain airgid a bhailiú leis an bhfadhb a réiteach. Sa bhliain 1912 scríobh sé chuig an dlíodóir Meiriceánach, John Quinn, ag lorg cabhrach uaidh. Bhí seisean tar éis turais W.B. Yeats agus Dhubhghlais de hÍde go Meiriceá a eagrú i dtosach na haoise, ach faoin am a ndearna an Piarsach teagmháil leis bhí sé tar éis éirí bréan de na hÉireannaigh ag teacht anall ag déircínteacht agus, mar a mhíníonn Úna Ní Bhroiméil ina haiste 'An Piarsach i Meiriceá,' 'shocraigh sé gan baint dá laghad a bheith aige le héilimh an Phiarsaigh.' Mhol sé don Phiarsach, áfach, 'dul chun cainte le John Devoy agus le Seumas MacManus' (219), rud a rinne sé. Dá thoradh sin, 'bhí dlúthnasc idir cuspóirí Devoy i leith [Óglaigh na hÉireann] agus misiún an Phiarsaigh,' ionas gur 'meascán d'ábhair chultúrtha agus ábhair náisiún[aío]cha pholaitiúla' (223) a bhí sna léachtaí a thug an Piarsach agus go ndeachaigh cuid den airgead a bailíodh chuig an Irish Republican Brotherbood a raibh Clan na Gael faoi cheannasaíocht an tsean-Fhínín Devoy nasctha leis ó 1877.

Sna litreacha a chuir a dheartháir Liam chuig Pádraig le linn na tréimhse a chaith sé i Meiriceáleag sé béim arís agus arís eile ar a thábhachtaí a bhí sé oiread airgid agus ab fhéidir a bhailiú, ach ar deireadh thiar ní bhfuair Pádraig dóthain leis na fiacha a ghlanadh. Bhí fonn air ina dhiaidh sin filleadh ar Mheiriceá, ach níor éirigh leis é sin a dhéanamh agus lean sé air ag coraíocht le fiacha na scoile fad a mhair sé.

Ba í an litríocht, a cineál, a tábhacht, a cleachtadh agus a hidéil (64) an dara hábhar mór a bhíodh idir chamáin ag an bPiarsach agus é ina eagarthóir ar $A n$ Claidheamb Soluis. Léiríonn Alan Titley ina aiste 'Gairm na Litríochta' gur den tábhacht é, dar leis an bPiarsach, a dhearbhú gurbh í litríocht na Gaeilge seachas an litríocht Angla-Éireannach fíorlitríocht na hÉireann, siúd is go raibh meas aige ar W.B. Yeats (65-68). Maidir le litríocht na Gaeilge, bíodh gurbh í an tseanlitríocht a chéadmhúscail an tsamhlaíocht ann, 'ba léir dó ... nach bhfónfadh [a leithéid] beag ná mór don aois nua' (63) agus d'áitigh sé i sraith de léirmheasanna in An Claidheamb Soluis i 1906 nár mhór don nualitríocht a bheith nua-aoiseach: 'We would have our literature modern not only [in] form ... but also in texture, tone and outlook. This is the twentieth century; and no literature can take root in the twentieth century which is not of the twentieth century'(Mac Piarais 1906).

Bhí an Piarsach ina scríbhneoir cruthaitheach chomh maith lena bheith ina chriticeoir agus foilsíodh cuid mhaith dá shaothar cruthaitheach ar An Claidheamb Soluis. Pléann Gearóid Denvir a chuid filíochta, scéalta agus drámaí Gaeilge, chomh maith le mórán gnéithe eile dá shaol agus dá shaothar, ina aiste fhairsing 'Ó Pheann an Phiarsaigh: Athléamh 
ar Shaothar Liteartha Gaeilge an Phiarsaigh.' Admhaíonn Denvir go raibh an Piarsach 'ar an eolas faoi staid réa[d]ach na Gaeltachta' (97), agus deir gur 'tearmann idéalach nó útóipe Gaelach,' 'domhan ... a bhfuil luachanna an spioraid faoi rath agus faoi mheas ann,' 'domhan na soineantachta neamhurchóidí,' 'Pobal cráifeach a chleachtas gan cheist creideamh cóir na hEaglaise ... faoi láimh chineálta a gcuid sagart den chuid is mó' (98-99) atá cruthaithe ag an bPiarsach sa chuid is mó dá ghearrscéalta. Áitíonn sé gur léiriú ar phobal agus ar shaol na Gaeltachta mar ba mhaith leis an bPiarsach iad a bheith atá san útóipe úd. Tá dath ar a áitiú, gan amhras, ach sílim nár mhór dó trácht ar na heisceachtaí, mar atá, an dá scéal inar chruthaigh an Piarsach diostóipe seachas útóipe.

Is é an scéilín 'An Deargadaol' an eisceacht is suntasaí, b’fhéidir. Más é an sagart 'mar phearsa shoilíosach gheanúil thuisceanach' (100) an t-idéal, is é a mhalairt sin glan atá sa sagart sa scéal sin agus is é is cionsiocair leis an uafás a ghintear nuair a bhaineann sé mí-úsáid as a údarás, ní hamháin chun bean bhocht a fhógairt go poiblí toisc go raibh peaca éigin déanta aici, ach chun a ordú don phobal gan aon bhaint a bheith acu léi. Údarás an Stáit seachas údarás na hEaglaise atá i gceist sa dara sampla, 'An Bhean Chaointe,' ina ndaortar fear óg i ndúnmharú nach raibh sé ciontach ann, ionas go dtéann a mháthair as a meabhair nuair a fhaigheann sé bás i bpríosún. Tá toradh léanmhar ar imeachtaí an dá scéal sin i bhfad na haimsire freisin: sa chéad chás tugann an reacaire le fios go gcreideann sé gur scriosadh a chlann agus é féin mar go ndeachaigh siad thar chros an tsagairt, agus sa dara cás léiríonn a ndeir an reacaire agus a mhac i ndeireadh an scéil go bhfuil íde na mná agus a mic tar éis iad a shaighdeadh chun ceannairce in aghaidh údarás an Stáit (féach Ó Háinle 2000).

Tagann mí-úsáid na cumhachta agus an údaráis i gceist sa dán 'A Mhic Bhig na gCleas' freisin. Sa chás seo, deir Denvir go bhfuil 'cailleadh na soineantachta ag an mbuachaill óg tríd an míghníomh a rinne sé' (94) ar cheann de dhá ábhar an dáin. Ach ní féidir do dhuine peaca a dhéanamh gan a thuiscint go bhfuil a bhfuil á dhéanamh aige ina pheaca. Nuair nár thuig an buachaill go raibh peaca á dhéanamh aige, ní raibh peaca á dhéanamh aige agus ní raibh an tsoineantacht caillte aige. Deir Denvir freisin gurb é an dara hábhar atá ag an dán maithiúnas 'tuisceanach grámhar an reacaire' d'éis an pheaca (94). Tá sé sin bunaithe aige ar an dá véarsa tosaigh den dán, agus fágann sé as an áireamh an chuid eile de mar a ndéantar tagairt don sásamh a bhain an cainteoir as an bpóg a thug sé don bhuachaill agus é á chur i gcomparáid aige leis an sásamh a bhain sé as caidreamh fisiciúil le mná. Is léir go bhfuil sásamh gnéasach bainte ag an reacaire as an bpóg ('An lasair id' ghnúis / De m'uamhan bheadh bán / Dá léifeá mo rúin. // An té 'gá bhfuil mo rúin, / Ní fiú é teagmháil leat'), rud a fhágann go bhfuil an bonn bainte ag a thruaillíocht féin ón údarás atá éilithe aige le duine a dhaoradh nó maithiúnas a thabhairt dó, mar go gcoilleann aon sórt ciontachta éileamh lucht an údaráis ar ghradam morálta (féach Ó Háinle 2000: 131-138).

Cuireann Denvir ar ár súile dúinn go bhfuil an bás mar théama i gcuid mhaith de dhánta an Phiarsaigh agus áitíonn go bhfáiltíonn sé, mar a d'fháiltigh na filí rómánsúla, 'roimh an mbás ... a thabharfas faoiseamh dó ó phianta an tsaoil agus ó bhuairt agus ó ennui na beatha daonna' (96). Maidir leis an dán 'Fornocht do Chonac Thú,' nochtann sé an bharúil spéisiúil go bhféadfaí an dán sin a léamh 'i gcomhthéacs thuiscint ... na Rómánsaíochta faoi dhosheachantacht an bháis seachas mar aisling pholaitiúil ag tuar a bháis féin san Éirí Amach' (96). Dar le Denvir gurb é an bás a shlánaíonn an laoch agus a mhuintir sa dráma $A n$ $R i$, ach nach mór don laoch óg a bheith saor ó chion (105). Ar an ábhar sin bhí ar an bPiarsach tréithe áirithe de chuid Chú Chulainn na Tána a scagadh as an léiriú a thug sé ar an laoch in Macghniombartha Chúchulainn, tréithe 'nach gcuirfeadh leis an íomhá idéalach den laoch a bhí sé ag iarraidh a chruthú agus a chur chun cinn' (108).

Más pobal idéalach an pobal Gaeltachta atá cruthaithe ag an bPiarsach sa chuid is mó dá ghearrscéalta, is léir ón dá eisceacht atá luaite agam agus ó chuid dá shaothar dioscúrsach nach raibh sé dall ar fhíorshaol mhuintir na Gaeltachta. Pléitear an taithí a bhí aige ar an saol sin in dhá aiste sa díolaim seo, mar atá, 'An Piarsach i Ros Muc'le Seosamh Ó Cuaig agus 'An Piarsach agus an Ghaeltacht: Coincheapú agus Bearta Praiticiúla' le Caitríona Ó Torna. Thug an Piarsach cuairteanna ar go leor ceantar Gaeltachta, ach is i Ros Muc a chuir sé an teach á thógáil inar chaith sé mórán ama ina dhiaidh sin. Tugann an Cuaigeach tuairisc bhreá ar an aithne a bhí ag a shin- 
seanathair, Seán Ó Gaora, agus ag a mhac siúd, Colm, ar an bPiarsach, ar an gcaidreamh a bhí aige leosan agus le daoine eile i Ros Muc agus ar an ngean a bhí aige orthu agus acu siúd airsean. Chuige sin baineann sé leas as leabhar Choilm, Mise (1943), agus as roinnt foinsí áitiúla eile. Bréagnaíonn sé an bharúil nár thug an Piarsach 'aird ar bith ar an mbochtaineacht a bhí timpeall air i Ros Muc' agus tugann tuairisc ar an mbaint a bhí aige le toscaireacht a chuaigh go Baile Átha Cliath d'fhonn cabhair a iarraidh le scéim a chur ar bun le comhairle a chur ar an bpobal a chuideodh leo teacht slán ón eitinn a bhí 'ag leagan úr agus críon le chéile ag an am' (209).

Maidir le scríbhinní dioscúrsacha an Phiarsaigh, déanann Caitríona Ó Torna idirdhealú idir na cinn a scríobhadh roimh 1903 (nuair a ceapadh é mar eagarthóir ar An Claidheamb Soluis) agus na cinn a scríobhadh ina dhiaidh sin. I mblianta a óige, dar léi, bhí sé faoi thionchar dhúchasóirí ghluaiseacht na hAthbheochana (177) agus a gcuid bolscaireachta a léirigh an Ghaeltacht 'mar thobar an dúchais, gabhdán suáilce, áis oidhreachtúil don tír go léir'(176) agus a thug cur síos idéalach útóipeach ar mhuintir na Gaeltachta 'mar phobal a bhí dílis don traidisiún Gaelach, pobal soineanta cráifeach, pobal a bhí sona in ainneoin na bochtaineachta, pobal díograiseach, dolcarnach, umhal' (177). Measann sí freisin gur féidir a áitiú nach amhlaidh go raibh neamhshuim á déanamh aige 'de chruatan agus de dheacrachtaí an tsaoil' sna ceantair Ghaeltachta ach 'gur leag sé béim ar chráifeacht, glaineacht, cineáltas agus flaithiúlacht' mhuintir na Gaeltachta d'fhonn 'léargas úr a thabhairt ar cheantar agus ar phobal nach raibh meas orthu le fada' (182). A luaithe a bhí sé ceaptha ina eagarthóir ar An Claidheamb Soluis deir Ó Torna go bhfeictear 'plé agus cáineadh agus tuairimíocht réadúil i dtaobh shaol iarbhír na Gaeltachta i saothar dioscúrs[ach] an Phiarsaigh' (184) agus 'an cainteoir dúchais Gaeilge á cheangal le hÉireannachas agus le náisiúnachas [náisiúntacht?]' (185). Eisceacht ghránna, dar liom, ab ea an aiste 'Gleó na gCath' inar thug sé "'traitor", "“fool”, "'weakling”" agus “"coward” ar an nGaeilgeoir dúchais a rachadh ar imirce ón nGaeltacht. Amhlántacht agus neamh-mhothálacht dhochreidte $a b$ ea é ráiteas mar sin a chur i gcló. Ní leor dar liom a rá, mar a deir Ó Torna, gur 'bhain géire neamhchoitianta' leis, fiú más amhlaidh gur léiriú é ar thuiscint an Phiarsaigh 'ar a thubaistí a bhí an imirce do thodhchaí na Gaeltachta, na Gaeilge agus na hÉireann trí chéile' (186). Os a choinne sin thall ní mór a admháil, mar a shonraíonn Ó Torna, gur iomaí moladh praiticiúil a rinne an Piarsach 'a mheas sé a chuirfeadh srian le nós na himirce' agus go dtugadh sé tacaíocht do scéimeanna den sórt sin a bhí á moladh ag Athbheochanóirí eile (186-187).

Ina haiste 'Smaointeoireacht an Phiarsaigh ar an Náisiúnachas agus ar an bPolaitíocht, anuas go dtí 1913,' luann Mary N. Harris gur áitigh an Piarsach i litir a scríobh sé chuig Tom Kettle i 1904 'go raibh gluaiseacht na teanga níos tábhachtaí ná an ghluaiseacht pholaitiúil, ó thaobh na náisiúntachta de' (247-248) agus gurbh é a bharúil i 1907 gurbh fhiú glacadh leis an leagan maolaithe den Rialtas Dúchais a bhí i gceist leis an mBille um Chomhairle na hÉireann 'ar mhaithe le smacht a bheith ag Éireannaigh ar chúrsaí oideachais' (248). De réir a chéile, áfach, thosaigh sé ag teacht ar mhalairt aigne. I mí an Mhárta 1912 bhunaigh sé an iris An Barr Buadh agus nuair a chuir sé an cumann polaitíochta 'Cumann na Saoirse' ar bun in Aibreán na bliana céanna sin, bhí sí mar ghuth ag an gcumann sin. I nGaeilge ar fad a bhí aistí na hirise úd agus ba é an Piarsach féin a scríobh tuairim is a leath díobh, idir fhilíocht agus phrós. Seachtanán a bhí inti ach, faraor, níor mhair sí ach trí mhí agus d'éag 'Cumann na Saoirse' thart ar an am céanna léi. Bhí sé de mhórthábhacht le $A n$ Barr Buadh gur thug sé an Piarsach agus Éamonn Ceannt le chéile (260). Radacach ab ea Ceannt mar is léir ó 'Fáth an Chumainn Nua,' aiste a d'fhoilsigh sé ar An Barr Buadh in Aibreán 1912 inar áitigh sé gur chóir do Ghaeil troid ar son na hÉireann agus go mbeadh orthu dul i dtaithí ar ghunnaí (254). Bhí an Piarsach níos measartha ach chonacthas dó anois, dar le Harris, 'gur deineadh faillí i gceist na saoirse i rith na mblianta' (254-255).

I Meitheamh 1913 thosaigh an Piarsach ag foilsiú na sraithe aistí dar teideal 'From a Hermitage' in Irish Freedom, nuachtán an Irish Republican Brotherhood, "with the deliberate intention," mar a dúirt sé féin ina dhiaidh sin, "'of goading those who shared my political views to commit themselves definitely to an armed movement" (259). Ar an 8 Samhain 1913 foilsíodh a aiste 'The Coming Revolution' inar áitigh sé go raibh gá le hairm agus gur ghá dul i dtaithí ar 
dhoirteadh fola (259-260), agus coicís ina dhiaidh sin bhí sé páirteach i mbunú Óglaigh na hÉireann. Tugann Harris léargas spéisiúil ar mheon an Phiarsaigh i dtaca le cúrsaí na mban (255-256 agus 263 nóta 39). I mí na Bealtaine 1908, thug sé "the more important half of Ireland's population"' (140) ar na mná agus 'fuair sé locht ar a laghad ban a bhí ar choiste rialaithe na hOllscoile Náisiúnta agus d'áitigh sé gur chóir go mbeadh deis ag mná ar ollúnachtaí a fháil' (255). I 1912, nuair a bhí feachtas ar bun ag an Irish Women's Franchise League chun ceart vótála a bhaint amach do mhná, mhol sé 'meanma na mban' (255) nuair a ionsaíodh grúpa díobh ar shráideanna Bhaile Átha Cliath i ndeireadh mhí Mhárta na bliana sin agus dúirt dá mbeadh cúig chéad fear chomh misniúil leo in Éirinn nach ' $\mathrm{i}$ dtaobh le dea-thoil reachtaire Ghallda a bheadh saoirse Ghael' (An Barr Buadh, 5 Aibreán 1912). Gheall sé, ach a mbeadh Éire saor, go mbeadh ceart vótála agus 'gach cead agus gach céim [acu] dá raibh ag bantracht Éireann' sa seanam (An Barr Buadh, 12 Aibreán 1912); bhí iontas air 'nár éisteadh leis na teachtairí a chuir na mná chun na Comhdhálà faoin Rialtas Dúchais (An Barr Buadh, 4 Bealtaine 1912); agus, i gceann de na hailt 'From a Hermitage' i mí Iúil 1913, cháin sé na fir a cheap gur éileamh áiféiseach $a b$ ea éileamh na mban ar cheart vótála. Luann Harris, áfach, gur chuir sé in iúl do na mná nár chóir an t-éileamh ar cheart vótála do mhná a cheangal leis an éileamh ar Rialtas Dúchais agus gur iarr sé orthu a nasc le suffragettes Shasana a bhriseadh (256).

Ina aiste siúd, 'I dtreo an Mhíleatachais agus na Poblachta: an Piarsach agus an Pholaitíocht, 19131916,' aontaíonn J.J. Lee le Harris i dtaobh thábhacht na n-aistí 'From a Heritage' agus 'The Coming Revolution' agus bhunú Óglaigh na hÉireann mar chéimeanna tábhachtacha ar bhóthar an Phiarsaigh ón náisiúnachas cultúrtha go dtí an poblachtachas agus an réabhlóid. Dar leis gurbh í aidhm an Phiarsaigh agus na haistí sin á scríobh aige 'dul i bhfeidhm ar cheannairí an IRB, ag léiriú go raibh an mianach ceart ann' ionas 'go bhfeicfeadh drong ceannais an IRB é mar réabhlóidí cruthanta a d'fhanfadh sa choimhlint go dtí an dé deiridh' (270-271,273). Má bhí na ceannairí sin amhrasach faoina dhindiúirí poblachtacha, bhí deis aige i mí na Samhna 1913 'chun a dhindiúirí mar fhear míleata a neartú' nuair a bunaíodh Óglaigh na
hÉireann agus go bhfuair sé 'ballraíocht agus gradam ... in eagraíocht a raibh struchtúr míleata aige.'I mí na Nollag 1913, fuair sé a mhian nuair a ligeadh isteach é san IRB (274).

Nuair a thosaigh an cogadh domhanda i 1914, mhol John Redmond do na hÓglaigh dul isteach in arm Shasana. Scoilteadh na hÓglaigh agus bhí tionchar níos mó ná riamh ag an IRB ar an bhfórsa beag a bhí fágtha. Mar a deir Lee, 'bhí baill ag an IRB in ionaid straitéiseacha sna hÓglaigh' agus 'ba é an Piarsach ba mhó ... a chuir lena thionchar san IRB agus sna hÓglaigh' (276) nuair a ceapadh é ina oifigeach preas agus ina stiúrthóir ar eagar míleata ag na hÓglaigh agus nuair a cheap an IRB i mBealtaine 1915 é mar bhall de choiste triúir a bhí chun na pleananna d’éirí amach a bhrú chun cinn agus a bheachtú. Faoi mhí Mheán Fómhair 1915 bhíothas tar éis é a thabhairt isteach in Ardchomhairle an IRB. Glacann Lee leis go raibh 'an phríomhpháirt ag an bPiarsach ... i ndráma na Cásca 1916' (278): ba é a ainmníodh mar Uachtarán ar Rialtas Sealadach na Poblachta, a léigh an Forógra lasmuigh d'Ard-Oifig an Phoist agus a chinn ar an Éirí Amach a thabhairt chun clabhsúir le géilleadh gan choinníoll (278-279). Deir sé gur iompair an Piarsach é féin go 'honórach' le linn an Éirí Amach agus 'go dínitiúil, cróga' os comhair na cúirte míleata agus gur ghlac sé leis an mbás 'go calma' (279). Theip ar an Éirí Amach, ach is cinnte go raibh an Piarsach sásta glacadh leis go raibh a chuid fola á híobairt aige mar íobairt shlánaithe ar son shaoirse na tíre. Ach áitíonn Lee go húdarásach nárbh é an t-éirí amach a tharla Seachtain na Cásca an t-éirí amach a bhí i gceist agus a bhí á phleanáil ag na ceannairí, agus an Piarsach ar dhuine acu, le cúpla bliain roimh 1916 (279-280): ní 'íobairt fola’ a bhí á beartú acu ach gníomh buach míleata.

San aiste dheiridh sa leabhar seo, 'Cáil an Phiarsaigh i ndiaidh a Bháis: Beathaisnéisí agus Breithiúnais,' pléann Gearóid Ó Tuathaigh na tuairiscí agus na breithiúnais atá tugtha ar an bPiarsach ón uair a cuireadh chun báis é i leith, agus an tionchar a bhí acu ar pholaiteoirí agus ar phobal na tíre. Is beag aithne a bhí ag an bpobal air sular cuireadh chun báis é; ach laistigh de bhliain i ndiaidh a bháis bhí foilsitheoirí ag iomaíocht le chéile ag iarraidh cnuasaigh dá chuid scríbhinní a fhoilsiú agus ag féachaint le scéal an Éirí Amach a mhíniú don saol mór. Ba é Desmond Ryan a 
d'fhoilsigh an chéad bheathaisnéis shubstainteach den Phiarsach, The Man called Pearse, i 1919. Deisceabal dílis de chuid an Phiarsaigh ab ea Ryan: iarscoláire de chuid Scoil Éanna ab ea é, agus teagascóir sa scoil sin ina dhiaidh sin; agus bhí sé páirteach san Éirí Amach agus in éineacht leis an bPiarsach in Ard-Oifig an Phoist. Ní nach ionadh, naomhsheanchas atá ina thuairisc ar an bPiarsach inar léirigh sé é mar 'laoch ionspioráideach ... fathach fir agus tírghráthóir [a bhí] uasal go smior' (288). A dhála sin, 'portráid de naomh - cuid mhaith' (288) a bhí in La vie de Patrice Pearse a d'fhoilsigh an náisiúnaí Briotánach Louis N. Le Roux i 1932, agus beathaisnéis bháúil a bhí in Pádraig Mac Piarais a d'fhoilsigh Séamus Ó Searcaigh i 1938. Bhí cáil an Phiarsaigh "cuisnithe" mar laochnaomh réabhlóid ghlórmhar na bliana 1916’ (290-291). Sa tréimhse sin, an t-aon tuairisc a foilsíodh inar cáineadh an Piarsach, Denis Gwynn, iarscoláire eile de chuid Scoil Éanna a chuaigh 'le saighdiúireacht sa Chéad Chogadh Mór in éide an Rí a scríobh í (289). Ina aiste 'Patrick Pearse' a foilsíodh ar Dublin Review i 1923, 'chuir sé ina leith gur bhain sé úsáid as Scoil Éanna chun buíon ógánach imprisiúnach [sic] a earcú dá thionscnamh réabhlóideach' agus d'fhógair sé go raibh “colossal egotism” ag baint leis an bPiarsach (289).

Beagán faoi bhun leathchéad bliain tar éis an Éirí Amach 'bhí athrú ag teacht ar na hinsintí a bhí á bhfoilsiú ar thréimhse na réabhlóide' (291). I sraith d'fhoilseacháin idir 1961 agus 1973 nocht F.X. Martin 'dearcadh i leith an Phiarsaigh ... a bhí fuaraigeanta agus criticiúil, muna raibh sé diúltach ar fad'; ina beathaisnéis Pádraig Pearse: A New Biography i 1966 fuair Hedley McCay lochtanna ar thuairimí an Phiarsaigh agus 'ar a chuid ráiteas faoin bhforéigean' (292); agus in The Imagination of an Insurrection i 1967 d'áitigh William I. Thompson 'go raibh an tsamhlaíocht fhileata (seachas an pholaitíocht phraiticiúil) go mór in uachtar' in aigne 'chuid de cheannairí an Éirí Amach, an Piarsach san áireamh' (293). Is léir go dtuigtear don Tuathach nach bhfuil na breithiúnais i gcuid de na saothair sin bunaithe ar aon taighde nua, murab ionann agus saothair le Breandán Mac Giolla Choille agus Pádraig Ó Snodaigh agus 'cnuasaigh éagsúla aistí' a foilsíodh i 1966 atá bunaithe ar thaighde nua agus atá 'níos meáite' (253). Spreag comóradh an leathchéid i 1966 agus roinnt cláir theilifíse díospóireacht i measc an phobail ar phearsantacht agus ar thréithe cheannairí an Éirí Amach.

Ansin sna seachtóidí tosaíodh ag foilsiú ábhar cáinteach faoin bPiarsach. In aiste dar teideal 'The Canon of Irish History: a Challenge' a foilsíodh i 1972, lochtaigh an tÍosánach, Francis J. Shaw, an traidisiún poblachtach go géar agus thug diamhasla ar thagairtí a rinne an Piarsach dá fhéiníobairt féin inar shamhlaigh sé í le bás Chríost ar an gcros; agus d'fhoilsigh Raymond J. Porter beathaisnéis ghairid, P.H. Pearse, i 1973 inar chuir sé "milleán faoi leith ar an bPiarsach as a chuid scríbhinní "buile" ag adhradh an fhoréigin agus na féiníobairte' (294-295). D’fhoilsigh Ruth Dudley Edwards Patrick Pearse: The Triumph of Failure i 1977, leabhar a raibh 'taighde láidir mar thaca' aige, ach a raibh 'nóta criticiúil [nó] cáinteach' ann. Spreag sé 'conspóid, go háirithe, faoi thuair[i]míocht Dudley Edwards ar an gclaonadh h[o]maighnéasach, dar léi, a bhí láidir ins an bPiarsach' (295-296). Ba í an bheathaisnéis sin 'an cuntas ba chuimsithí agus ba dhúshlánaí dár scríobhadh ar an bPiarsach go dtí sin,' dar leis an Tuathach. 'Ba ghéire ar fad (agus ba bhinbí) an cáineadh ar an bPiarsach a dhein Xavier Carty ina leabhar In Bloody Protest: The Tragedy of Patrick Pearse' a foilsíodh i 1978 (296).

Faoi lár na seachtóidí bhí an choimhlint fhuilteach i dtuaisceart na hÉireann faoi lán seoil agus ba dheacair, dar leis an Tuathach, d'údair na leabhar úd éalú óna scáth. D'éirigh ceannairí polaitíochta, iriseoirí agus craoltóirí, agus an mórphobal seachantach ar chaint faoin Éirí Amach agus ar chomóradh a dhéanamh air. I 1979, áfach, rinneadh cothrom an lae a rugadh an Piarsach a chomóradh ar bhealaí éagsúla, agus d'fhoilsigh an scoláire oideachais, Séamas Ó Buachalla, bailiúcháin thábhachtacha de scríbhinní an Phiarsaigh an bhliain sin agus an bhliain dár gcionn (298-299). Sna hochtóidí agus anuas go lár na nóchaidí foilsíodh 'aistí agus corrleabhar ina ndearnadh iarracht gnéithe faoi leith de smaointe an Phiarsaigh agus dá phearsantacht a iniúchadh go grinn.' I gceann de na leabhair sin, Patrick Pearse and the Lost Republican Ideal a tháinig amach i 1991, rinne Brian P. Murphy 'cosaint láidir ar an bPiarsach' agus é 'ag míniú na $[\mathrm{g}]$ cúinsí áirithe staire sna blianta 1914-1916 faoinar ghaibh sé leis an bpoblachtachas míleata' (300). Deir an Tuathach go raibh 'an aeráid idé-eolaíoch agus an 
comhthéacs polaitíochta ag athrú in Éirinn' faoi thús na mílaoise nua agus go raibh foinsí nua eolais ag teacht chun solais, 'go háirithe an fhianaise ar imeachtaí Sheachtain na Cásca a thug Óglaigh a throid san Éirí Amach don Bureau of Military History,' ionas gurbh fhéidir 'saol agus ról an Phiarsaigh a mheas ar bhealach níos cothroime' (300-301), rud a rinneadh in The Life and After-Life of P.H. Pearse / Pádraig Mac Piarais: Saol agus Oidhreacht (eag. Roisín Higgins agus Regina Uí Chollatáin, 2009), san 'aiste mheáite' 'Patrick Henry Pearse 1879-1916' in Dictionary of Irish Biography (eag. James McGuire agus James Quinn, 2009) le J.J. Lee, údar na haiste leathdheiridh sa leabhar atá á léirmheas anseo, agus sa bheathaisnéis leis an scoláire Dúitseach, Joost Augusteijn, Patrick Pearse: The Making of a Revolutionary (2010), atá ar an leabhar is minice dá ndéantar tagairt sa leabhar seo.

Easnamh mór amháin atá ar An Piarsach agus 1916, go áirithe mar chuimhneachán ar Éirí Amach na Cásca 1916, nach bhfuil aon aiste ann faoi pháirt an Phiarsaigh san Éirí Amach sin. Is fíor go ndéantar roinnt mhaith tagairtí do ghnéithe di sin in aistí éagsúla sa leabhar, ach ba dhóigh liom gur thuill an chuid deiridh úd dá shaol go ndéanfaí cur síos agus plé comhtháite ar leith uirthi.

Tá an leabhar seo an-slachtmhar agus ar bheagán dearmad cló. Ní mór dom a rá, áfach, go bhfuil riar nach beag ann d'abairtí agus de fhrásaí máchaileacha, mar shampla:

- 'an iliomad réimse spéiseanna' (18);

- 'ceanglaíonn sé Parnell mar chomharba Tone' (51);

- 'chun an difríocht idir scríbhinní luatha na nualitríochta [agus X?] a mheas' (51);

- 'an ceathrar "taibhsí" a raibh tionchar chomh mór sin [acu] air' (51);

- 'léiríonn na scríbhinní seo go raibh spleáchas áirithe ar fhóram na hiriseoireachta don Phiarsach' (54);

- 'bhí dhá aidhm acu. An chéad cheann díobh ba ea an Ghaeilge a chaomhnú ... agus ar labhairt na teanga a leathnú (60);

- 'saol nach bhfuil éalú uaidh murarbh is fáinleoga an scéil' (74);

- 'léirítear ... nárbh aon sifíní Éireannacha amháin a bhí ag séideadh faoin bPiarsach' (76);
- 'is iad liodán na laoch a léiríos' (90);

- '... John Wilson Foster a d'áitigh.' (97);

- 'i gceann d'ailt oideachais' (161);

- 'an constráidiú idéalach a shainítear go háirithe le dúchasóirî́ (185);

- 'Ba chóir go mbeadh airm ag Gaeil agus bheith ullamh' (252).

Feictear dom gurbh fhéidir gur tharla na botúin sin agus a leithéidí eile toisc nár caitheadh go leor cúraim le coigeartú téacsanna a scríobhadh don raidió nuair a bhí siad á réiteach le foilsiú.

Is maith ann an nóta beathaisnéise (307-308) a dhréacht Ciarán Ó Coigligh agus an Leabharliosta Roghnaithe (309-316) ina bhfuil an chuid is mó go mór fada de na leabhair dá ndéantar tagairt sna haistí curtha i dtaifead.

\author{
Cathal Ó Háinle \\ Coláiste na Trionóide, Baile Átha Cliath
}




\section{SAOTHAIR A CEADAÍODH}

Mac Piarais, P. (1906) 'About Literature'. In: An Claidheamb Soluis, 26 Bealtaine 1906.

Mac Piarais, P. (1912) 'An Chúis dá Plé. In: An Barr Buadh, 5 Aibreán 1912.

Mac Piarais, P. (1912) 'An Chúis dá Plé'. In: An Barr Buadh, 12 Aibreán 1912.

Mac Piarais, P. (1912) 'An Chúis dá Plé. In: An Barr Buadh, 5 Bealtaine 1912.

Ó Cathasaigh, A. (eag.) (2012) An Barr Bua. Baile Átha Cliath: Coiscéim.

Ó Háinle, C. (2000) 'An Piarsach: Misteachas, Moráltas agus Miotas'. In: Léachtai Cholm Cille 30: $125-131$.

Ryan, D. (1919) The Man Called Pearse. Dublin: Maunsel. 